\title{
Исследование методом спектроскопии фотоотражения слоев LT-GaAs, выращенных на подложках Si и GaAs
}

\author{
(С) Л.П. Авакянц ${ }^{1}$, П.Ю. Боков ${ }^{1,}$, И.П. Казаков ${ }^{2}$, М.А. Базалевский ${ }^{2}$, \\ П.М. Деев ${ }^{1}$, А.В. Червяков ${ }^{1}$ \\ ${ }^{1}$ Московский государственный университет им. М.В. Ломоносова \\ (фризический фракультет), \\ 119991 Москва, Россия \\ ${ }^{2}$ Физический институт им. П.Н. Лебедева Российской академии наук, \\ 119991 Москва, Россия \\ ฯ E-mail: pavel_bokov@physics.msu.ru
}

(Получена 8 июня 2017 г. Принята к печати 19 июня 2017 г.)

\begin{abstract}
Методом спектроскопии фотоотражения исследованы механические напряжения и плотности зарядовых состояний слоев LT-GaAs (LT - low temperature), выращенных методом молекулярно-пучковой эпитаксии на подложках $\mathrm{Si}(100)$ и $\mathrm{GaAs}(100)$. В спектрах фотоотражения структур $\mathrm{Si} / \mathrm{LT}-\mathrm{GaAs}$ присутствуют линии от фундаментального перехода GaAs $\left(E_{g}\right)$ в окрестности значения энергии 1.37 эВ, связанные с переходом между дном зоны проводимости и спин-орбитально отщепленной подзоной валентной зоны $\left(E_{g}+\Delta_{S O}\right)$ при 1.82 эВ. Обнаружено, что линия $E_{g}$ сдвинута в область меньших энергий, а линия $E_{g}+\Delta_{S o}-$ в область больших энергий в сравнении с соответствующими линиями спектров образцов GaAs/LT-GaAs. Cравнительные исследования спектров Si/LT-GaAs и GaAs/LT-GaAs позволили оценить механические напряжения в слоях LT-GaAs, выращенных на Si (по величине сдвига спектральных линий) и плотность зарядовых состояний на гетероинтерфейсе $\mathrm{GaAs} / \mathrm{Si}$ (по периоду осцилляций Франца-Келдыша).
\end{abstract}

DOI: 10.21883/FTP.2018.07.46039.8667

\section{1. Введение}

Арсенид галлия, выращенный при низких температуpax — LT-GaAs (LT - low temperature), является одним из перспективных материалов для создания монолитноинтегрированных элементов оптической связи в кремниевых интегральных схемах и терагерцовой техники [1], поэтому исследования его оптических свойств представляют значительный интерес.

Исследование оптических свойств слоев LT-GaAs на $\mathrm{Si}$ методами спектроскопии поглощения или отражения сопряжено с определенными трудностями из-за невысокого кристаллического совершенства, связанного с дислокациями несоответствия, преципитатами As и термическими напряжениями [2,3], приводящими к уширению спектральных линий. Для исследований полупроводниковых структур со слабовыраженными особенностями в спектрах поглощения или отражения используют методы модуляционной спектроскопии, в числе которых метод спектроскопии фотоотражения (ФО) [4]. Авторам не известны опубликованные результаты применения метода спектроскопии ФО к исследованию в структуре Si/LT-GaAs.

В данной работе приведены результаты исследования методом спектроскопии ФО деформаций и плотности зарядовых состояний слоев LT-GaAs, выращенных при низких температурах методом молекулярно-пучковой эпитаксии (МПЭ) на арсенид галлиевых и кремниевых подложках с ориентацией поверхности (100).

\section{2. Образцы и методика эксперимента}

Для исследования была выбрана серия образцов LT-GaAs, выращенных на подложках кремния (образцы 481 и 505) и арсенида галлия (образцы 480 и 544).

Слои LT-GaAs выращивались методом молекулярнолучевой эпитаксии (МЛЭ) на установке ЦНА-25. При изготовлении образца 481 перед загрузкой в установку МПЭ поверхность подложки $\mathrm{Si}(100)$ травилась химически в смеси $\mathrm{H}_{2} \mathrm{SO}_{4}: \mathrm{H}_{2} \mathrm{O}_{2}: \mathrm{H}_{2} \mathrm{O}(3: 1: 1)$, а затем пассивировалась в смеси $\mathrm{HF}: \mathrm{H}_{2} \mathrm{O}(1: 6)$ [4]. В ростовой камере проводился отжиг подложки $\mathrm{Si}$ в парах $\mathrm{As}_{4}$ при температуре $T_{s}=700^{\circ} \mathrm{C}$ в течение 10 мин. Затем температура $T_{s}$ опускалась до $270^{\circ} \mathrm{C}$, и выращивался слой LT-GaAs толщиной 0.5 мкм со скоростью $0.18 \mathrm{HM} / \mathrm{c}$ при эквивалентных давлениях паров $\mathrm{Ga}-3 \cdot 10^{-7} \mathrm{~T}$ и As $-5 \cdot 10^{-6} \mathrm{~T}$. После окончания роста температура $T_{s}$ повышалась со скоростью $0.2^{\circ} \mathrm{C} / \mathrm{c}$, и производился отжиг структуры при температуре $T=600^{\circ} \mathrm{C}$ в течение 6 мин.

При изготовлении образца 505 использовалась подложка $\mathrm{Si}(100)$ с отклонением на $4^{\circ}$ в направлении (011), которая не подвергалась химической очистке. В установке „Катунь“ производилась десорбция естественного окисла в парах $\mathrm{Si}$ при $T_{s}=850^{\circ} \mathrm{C}$, а затем наращивался буферный слой $\mathrm{Si}$ толщиной 200 нм. После остывания наносился слой $\mathrm{Sb}$, который предохранял поверхность $\mathrm{Si}$ от окисления при переносе образца в установку ЦНА-25. Затравливание слоя LT-GaAs производилось при $T_{s}=270^{\circ} \mathrm{C}$ импульсным осаждением по $1-2$ моно- 
слоя до толщины $\sim 3$ нм. Далее, при той же величине $T_{s}$, выращивался слой LT-GaAs толщиной 0.5 мкм и производился отжиг структуры при температуре $T_{s}=600^{\circ} \mathrm{C}$ в течение 10 мин.

Контрольные образцы 480 LT-GaAs и 544 LT-GaAs выращивались на подложке $\operatorname{GaAs}(100)$. После обычной процедуры десорбции окисла в образце 480 выращивался буферный слой $\mathrm{GaAs}$ при $T_{s}=580^{\circ} \mathrm{C}$ толщиной 200 нм, а затем - слой LT-GaAs при $T_{s}=270^{\circ} \mathrm{C}$ толщиной 0.5 мкм. Отжиг образца при повышенной температуре не проводился. Образец 544 выращивался аналогично, но толщина слоя LT-GaAs была 1 мкм, а после роста проводился отжиг при $T_{s}=600^{\circ} \mathrm{C}$ в течение 10 мин.

Спектры ФО регистрировались на автоматизированной установке с использованием двойного монохроматора [5]. В качестве источника зондирующего излучения использовалось излучение лампы накаливания (мощность 50 Вт). В качестве модулирующего - линия 532 нм DPSS-лазера (мощность 7 мВт) или $633 \mathrm{Hм}$ $\mathrm{He}-\mathrm{Ne}$-лазера (мощность 7 мВт). Излучение с разными длинами волн применялось для зондирования по толщине структуры. Частота модуляции составляла 500 Гц. Ширина аппаратной функции спектрометра не превышала 1 мэВ. Регистрация спектров ФО осуществлялась при комнатной температуре.

\section{3. Результаты и обсуждение}

Спектры ФО исследуемых образцов приведены на рис. 1. Видно, что амплитуда сигнала ФО образцов 480 и 544 (выращены на GaAs) больше, а ширина линий ФО меньше в сравнении со спектрами образцов 481 и 505 (выращены на $\mathrm{Si}$ ). Меньшая амплитуда и бо́льшая ширина линии ФО свидетельствуют о меньшем структурном совершенстве слоев Si/LT-GaAs. Во всех спектрах присутствуют линии в области энергий $1.37-1.43$ эВ $\left(E_{g}\right)$,

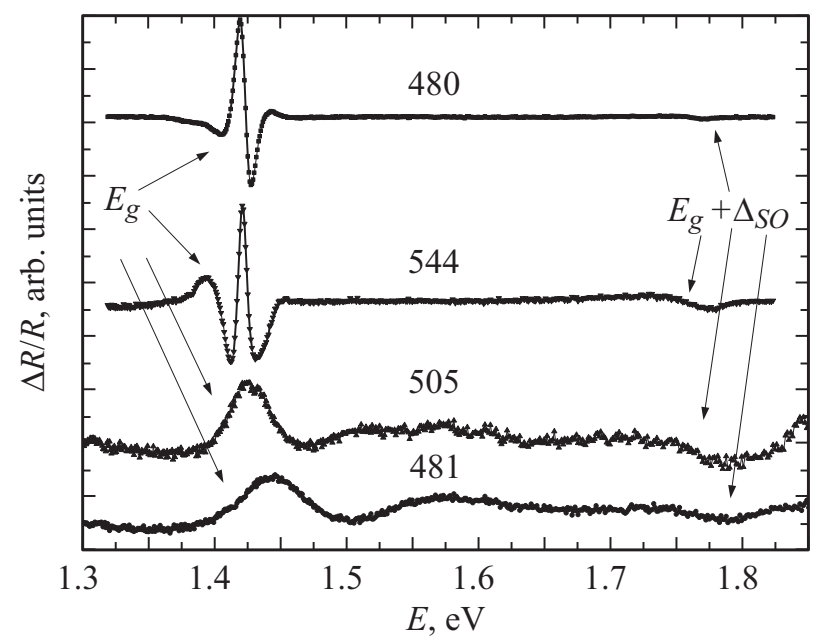

Рис. 1. Спектры ФО образцов 480 (LT-GaAs/GaAs), 544 (LT-GaAs/GaAs), 505, 481 (LT-GaAs/Si), зарегистрированные при модуляции излучением на длине волны 532 нм. связанные с фундаментальным переходом GaAs, и линии в области энергий $1.7-1.8$ эВ $\left(E_{g}+\Delta_{S O}\right)$, связанные с переходом между дном зоны проводимости и спинорбитально отщепленной подзоной валентной зоны. При этом для образцов 481 и 505, выращенных на кремнии, амплитуда линий $E_{g}+\Delta_{S O}$ сопоставима с амплитудой линий $E_{g}$.

\section{1. Деформационно-индуцированные изменения в спектрах ФО}

Линия ФО в области фундаментального перехода $E_{g}$ образцов 481 и 505 представляет собой осцилляции Франца-Келдыша. Для таких спектров существует простая асимптотика [6]:

$$
\frac{\Delta R}{R}(E) \propto \cos \left[\frac{2}{3}\left(\frac{\hbar \omega-E_{g}}{\hbar \Omega}\right)^{3 / 2}+\frac{\pi(d-1)}{4}\right],
$$

где $\hbar \omega-$ энергия зондирующего излучения, $E_{g}-$ энергия фундаментального перехода, $\hbar \omega-$ электрооптическая энергия:

$$
\hbar \Omega=\left(\frac{e^{2} E_{S}^{2} \hbar^{2}}{8 \mu}\right)^{1 / 3} .
$$

Здесь $\mu-$ приведенная межзонная эффективная масса, $E_{s}$ - напряженность встроенного электрического поля полупроводника, $d-$ размерность критической точки (в рассматриваемом случае $(d=3))$. Положения экстремумов осцилляций Франца-Келдыша $(\hbar \omega)_{j}$ даются выражением

$$
(\hbar \omega)_{j}=\hbar \Omega\left(F_{j}\right)+E_{g}, \quad j=1,2,3,
$$

где

$$
F_{j}=\left[3 \pi \frac{j-1 / 2}{2}\right]^{2 / 3} .
$$

Как видно из $(1)-(4)$, зависимость $(\hbar \omega)_{j}$ от $F_{j}$ представляет собой прямую с наклоном $\hbar \Omega$ и точкой пересечения оси ординат, равной $E_{g}$. Для образцов 481 и 505 было сделано построение зависимости $(\hbar \omega)_{j}$ от $F_{j}$. Результат построения приведен на рис. 2. МНК-анализ графика показывает, что линия $E_{g}$ сдвинута в область меньших энергий, до 1.36 эВ.

Линия в области 1.82 эВ представляет собой структуру с двумя экстремумами разных знаков, такие линии ФО принято описывать в рамках модели Аспнеса [7]:

$$
\frac{\Delta R}{R}(E)=\operatorname{Re}\left(A e^{i \varphi}\left(E-E_{0}+i \Gamma\right)^{-n}\right),
$$

где $E_{0}$ - энергия перехода, $\Gamma-$ феноменологический параметр уширения, $A$ и $\varphi-$ амплитудный и фазовый параметры соответственно, $E$ - энергия зондирующего излучения, $n$ - параметр, определяемый видом критической точки, в окрестности которой осуществляется электронно-дырочный переход (в нашем случае $n=2[7])$. 


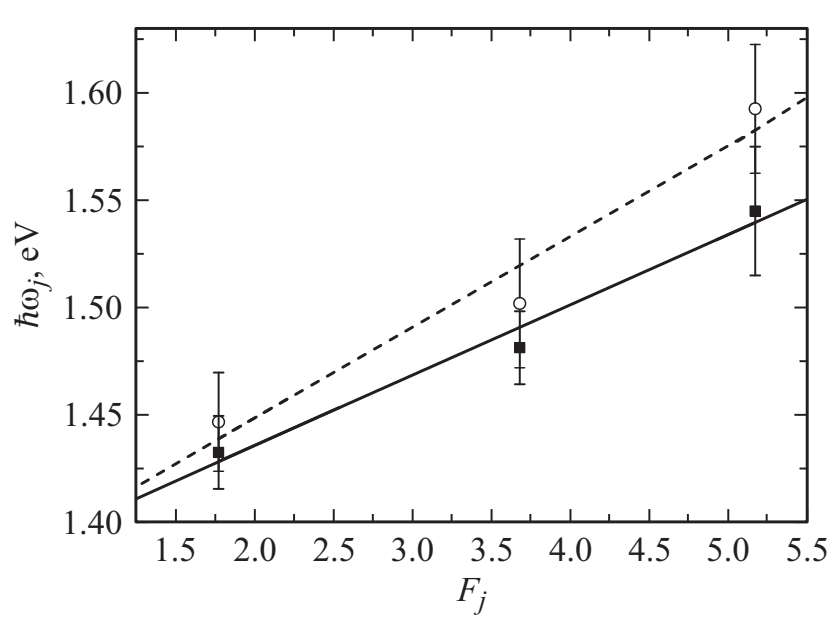

Рис. 2. Зависимость $\hbar \omega_{j}\left(F_{j}\right)$, круги - образец 481 , квадраты - образец 505, прямые - результат МНК-аппроксимации.

Из МНК-аппроксимации в рамках модели Аспнеса обнаружено, что линия $E_{g}+\Delta_{S O}$ сдвинута в область больших энергий, до 1.81 эВ.

Указанные сдвиги линий характерны для слоев GaAs, подверженных деформациям сжатия [8,9]. Деформационно-индуцированные сдвиги зоны проводимости $\Delta E_{c}$ и подзон тяжелых $\Delta E_{v l}$ и легких $\Delta E_{h l}$ дырок валентной зоны в центре зоны Бриллюэна определяются из соотношений $[8,9]$ :

$$
\begin{gathered}
\varepsilon=\frac{a_{1}-a_{2}}{a_{1}}, \\
\Delta\left(E_{g}\right)=A_{1} \varepsilon \\
\Delta\left(E_{g}+\Delta_{S O}\right)=A_{3} \varepsilon \\
A_{1}=-2 a \frac{C_{11}-C_{12}}{C_{11}}+b \frac{C_{11}+2 C_{12}}{C_{11}}, \\
A_{3}=-2 a \frac{C_{11}-C_{12}}{C_{11}} .
\end{gathered}
$$

Здесь $\varepsilon-$ относительная деформация слоя, $a_{1}, a_{2}-$ постоянные кристаллической решетки слоев $\mathrm{GaAs}$ и $\mathrm{Si}$, $a$ - гидростатический деформационный потенциал для зоны проводимости, $b$ - потенциал одноосных деформаций, $C_{11}, C_{12}$ - упругие постоянные. Используемые в моделировании параметры приведены в таблице.

В рамках модели (6)-(10) с учетом наблюдаемых сдвигов спектральных линий оценены относительные деформации слоев LT-GaAs. Они составили $\varepsilon\left(E_{g}+\Delta_{S O}\right)=0.0085 \pm 0.0017, \varepsilon\left(E_{g}\right)=0.0066 \pm 0.0011$

$$
\begin{array}{c|c|c|c|c|c}
\hline a_{1}, \AA(\mathrm{GaAs}) & a_{2}, \AA(\mathrm{Si}) & a_{v},{ }_{\text {эВ }} & b,{ }_{\text {эВ }} & C_{11}, \text { Па } & C_{12}, \text { Па } \\
\hline 5.6533 & 5.4311 & -8.3 & -1.7 & 11.9 \cdot 10^{11} & 5.38 \cdot 10^{11}
\end{array}
$$

Примечание. Параметры, используемые нами в моделировании (6)-(10), взяты из работы [13]. для образца 481 и $\varepsilon\left(E_{g}+\Delta_{S O}\right)=0.0086 \pm 0.0017$, $\varepsilon\left(E_{g}\right)=0.0065 \pm 0.0011$ для образца 505 , что коррелирует с литературными данными [10].

\section{2. Плотность поверхностных состояний на гетероинтерфейсе $\mathrm{GaAs} / \mathrm{Si}$}

Наклон зависимостей $(\hbar \omega)_{j}$ от $F_{j}$ (рис. 2) позволил оценить значения электрооптической энергии и напряженности встроенных электрических полей в образцах. Последние составили $(0.21 \pm 0.07) \mathrm{MB} / \mathrm{cm}$ для образца 481 и $(0.14 \pm 0.03) \mathrm{MB} /$ см для образца 505.

Для установления источника сигнала ФО с осцилляциями была проведена регистрация спектров при модуляции излучением DPSS- и $\mathrm{He}-\mathrm{Ne}$-лазеров на длинах волн 532 и 633 нм соответственно. В качестве примера на рис. 3 приведены спектры ФО образца 505. Видно, что модуляции на большей длине волны (т.е. с большей глубиной проникновения в образец) соответствуют спектры с большей амплитудой сигнала ФО в области фундаментального перехода. Различие амплитуд сигналов ФО указывает на то, что его источником является область гетероперехода $\mathrm{GaAs}-\mathrm{Si}$ - область, в которой возникают механические напряжения, обусловленные различием постоянных решетки [10].

Источником электрического поля в структурах 481 и 505 являются, по-видимому, заряды, сформированные в результате взаимного легирования гетероперехода Si-GaAs при отжиге. O таком взаимном легировании упоминается, например, в работе по вольтфарадному профилированию структур типа $\mathrm{GaAs} / \mathrm{Ge}$ [11] и GaAs/InGaAs [12]. Как и в [11,12], можно предположить, что взаимное легирование происходит на толщинах в несколько постоянных решетки, т. е. оно аналогично $\delta$-легированию. Согласно электростатической теореме Гаусса, по напряженности электростатического

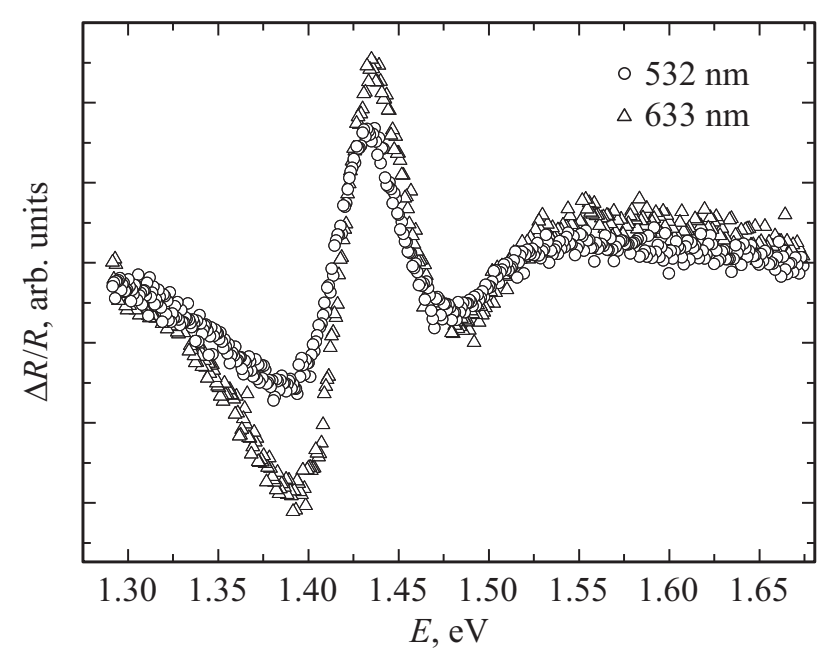

Рис. 3. Спектры ФО образца 505, полученные при модуляции излучением с длинами волн 532 нм (круги) и 633 нм (треугольники). 
поля можно оценить поверхностную плотность зарядов $\sigma$ на гетеропереходе:

$$
\sigma=2 \varepsilon \varepsilon_{0} E_{s},
$$

где $\varepsilon=12.6$ - статическая диэлектрическая постоянная $\mathrm{GaAs}, \varepsilon_{0}$ - электрическая постоянная [13]. Для образцов 481 и 505 поверхностная плотность зарядов на гетероинтерфейсе составила $(2.9 \pm 0.9) \cdot 10^{12}$ и $(2.0 \pm 0.4) \cdot 10^{12} \mathrm{~cm}^{-2}$ соответственно.

Таким образом, в случае образца 505, для которого применялась технология импульсного роста на начальном этапе, и механические напряжения, и поверхностная плотность зарядов на гетероинтерфейсе $\mathrm{GaAs}-\mathrm{Si}$ оказываются меньшими в сравнении с образцом 481. Метод спектроскопии ФО оказывается чувствительным к различию процессов роста и последующего отжига образцов Si/LT-GaAs.

\section{4. Заключение}

Методом спектроскопии ФО исследованы структуры LT-GaAs, выращенные при низких температурах на подложках кремния и арсенида галлия. В спектрах ФО слоев LT-GaAs/Si обнаружены осцилляции Франца-Келдыша в области фундаментального перехода $E_{g}$ и сигнал от $E_{g}+\Delta_{S O}$. Показано, что источником сигнала ФО является область гетероинтерфейса LT-GaAs/Si.

По периоду осцилляций Франца-Келдыша определены напряженности электрических полей в образцах 481 и 505. Они составили соответственно $(0.14 \pm 0.03)$ и $(0.21 \pm 0.07) \mathrm{MB} / \mathrm{cm}$. С использованием электростатической теоремы Гаусса оценены поверхностные плотности зарядов, они составили $(2.9 \pm 0.9) \cdot 10^{12}$ и $(2.0 \pm 0.4) \cdot 10^{12} \mathrm{~cm}^{-2}$.

Деформационно-индуцированные сдвиги линий $E_{g}$ и $E_{g}+\Delta_{S O}$ в спектрах ФО соответствуют деформациям сжатия GaAs. Оцененные значения относительных деформаций составили $\varepsilon\left(E_{g}+\Delta_{S O}\right)=0.0085 \pm 0.0017$, $\varepsilon\left(E_{g}\right)=0.0066 \pm 0.0011$ для образца 481 и $\varepsilon\left(E_{g}+\Delta_{S O}\right)=0.0086 \pm 0.0017, \varepsilon\left(E_{g}\right)=0.0065 \pm 0.0011$ для образца 505.

Таким образом, в случае образца 505, для которого применялась технология импульсного роста на начальном этапе, механические напряжения и поверхностная плотность зарядов на гетеропереходе оказываются меньшими. Это свидетельствует о большем структурном совершенстве образца 505 в сравнении с образцом 481.

Работа выполнена при частичной поддержке фонда РФФИ (гранты № 18-0200842 и № 16-29-03352 офи-м).

\section{Список литературы}

[1] Д.В. Лаврухин, А.Э. Ячменев, А.С. Бугаев, Г.Б. Галиев, Е.А. Климов, Р.А. Хабибуллин, Д.С. Пономарев, П.П. Мальцев. ФТП, 49, 932 (2015).
[2] Д.А. Винокуров, В.М. Лантратов, М.А. Синицын, В.П Улин, Н.Н Фалеев, О.М. Федорова, Я.Л Шайович, Б.С Явич. ФТП, 25, 1022 (1991).

[3] Wu-Yih Uen, T. Ohori, T. Nishinaga. J. Cryst. Growth, 156, 133 (1995).

[4] J. Misiewicz, P. Sitarek, G. Sek, R. Kudrawiec. Mater. Sci., 21, 263 (2003).

[5] Л.П. Авакянц, П.Ю. Боков, А.В. Червяков. ЖТФ, 75, 66 (2005).

[6] D.E. Aspnes, A.A. Studna. Phys. Rev. B, 7, 4605 (1973).

[7] D.E. Aspnes. Surf. Sci., 37, 418 (1973).

[8] X. Zhang, K. Onabe, Y. Nitta, B. Zhang, S. Fukatsu, Y. Shraki, R. Ito. Jpn. J. Appl. Phys., 30, L1631 (1991).

[9] Л.П. Авакянц, П.Ю. Боков, Т.П. Колмакова, А.В. Червяков. ФТП, 38, 1429 (2004).

[10] Р.В. Кузьменко, А.В. Ганжа, О.В. Бочурова, Э.П. Домашевская, Й. Шрайбер, С. Хильдебрандт, Ш. Мо, Э. Пайнер, А. Шлахетцкий. ФТП, 34, 73 (2000).

[11] M.K. Hudait, S.B. Krupanidhi. J. Vac. Sci. Technol. B, 17, 1003 (1999).

[12] А.Н. Петровская, В.И. Зубков. ФТП, 43, 1368 (2009).

[13] S.C. Jain, M. Willander, H. Maes. Semicond. Sci. Technol., 11, 641 (1996).

Редактор Г.А. Оганесян

\section{Photoreflectance study of LT-GaAs layers grown on $\mathrm{Si}$ and GaAs}

\section{L.P. Avakyants ${ }^{1}$, P.Yu. Bokov' ${ }^{1}$, I.P. Kazakov',} M.A. Bazalevsky ${ }^{2}$, P.M. Deev ${ }^{1}$, A.V. Chervyakov ${ }^{1}$

${ }^{1}$ Faculty of Physics Moscow State University,

119991 Moscow, Russia

${ }^{2}$ Lebedev Physical Institute

Russian Academy of the Sciences,

119991 Moscow, Russia

Abstract The mechanical stresses and densities of the charge states in LT-GaAs (LT - low temperature) layers grown by molecular-beam epitaxy on $\mathrm{Si}(100)$ and $\mathrm{GaAs}(100)$ substrates have been studied by photoreflectance. In the photoreflectance spectra of the GaAs grown on $\mathrm{Si}$ are presented lines from the $E_{g}$ of $\mathrm{GaAs}$ at value $1.37 \mathrm{eV}$ and from $E_{g}+\Delta_{S O}$ at value of $1.82 \mathrm{eV}$. These lines are shifted due to mechanical stresses. Comparative studies of the $\mathrm{Si} / \mathrm{LT}-\mathrm{GaAs}$ and $\mathrm{GaAs} / \mathrm{LT}-\mathrm{GaAs}$ allow to estimate the mechanical stresses in LT-GaAs layers grown on $\mathrm{Si}$ (in terms of the spectral lines shift) and the density of charge states on the $\mathrm{GaAs} / \mathrm{Si}$ heterointerface (through of the period of Franz-Keldysh oscillations). 